Siam J. Math. Anal.

Vol. 26, No. 1, pp. 21-34, January 1995

\title{
SLOW MOTION IN ONE-DIMENSIONAL CAHN-MORRAL SYSTEMS
}

\author{
CHRISTOPHER P. GRANT*
}

\begin{abstract}
In this paper we study one-dimensional Cahn-Morral systems, which are the multicomponent analogues of the Cahn-Hilliard model for phase separation and coarsening in binary mixtures. In particular, we examine solutions that start with initial data close to the preferred phases except at finitely many transition points where the data has sharp transition layers, and we show that such solutions may evolve exponentially slowly; i.e., if $\varepsilon$ is the interaction length then there exists a constant $C$ such that in $\exp (C / \varepsilon)$ units of time the change in such a solution is $o(1)$. This corresponds to extremely slow coarsening of a multicomponent mixture after it has undergone fine-grained decomposition.
\end{abstract}

Key words. Cahn-Hilliard equation, phase separation, transition layers, metastability

AMS subject classifications. 35B30, 35B25, 35K55

1. Introduction. One of the leading continuum models for the dynamics of phase separation and coarsening in a binary mixture is the Cahn-Hilliard equation, which in the one-dimensional case can be written as

$$
\begin{gathered}
u_{t}=\left(-\varepsilon^{2} u_{x x}+W^{\prime}(u)\right)_{x x}, \quad x \in(0,1) \\
u_{x}=u_{x x x}=0,
\end{gathered}
$$

Here $W$ represents the bulk free energy density as a function of the concentration $u$ of one of the two components of the mixture. (If, as is typically assumed, the total concentration of the mixture is a constant then the concentration of the second is determined by the concentration of the first.) The parameter $\varepsilon$ represents an interaction length and is assumed to be a small positive constant. This equation was derived in [8] based on the free energy functional of van der Waals [29]

$$
\mathcal{E}_{\varepsilon}[u] \equiv \int_{0}^{1}\left(W(u)+\frac{\varepsilon^{2}}{2}\left|u_{x}\right|^{2}\right) d x .
$$

We will usually work with the scaled energy $E_{\varepsilon}[u] \equiv \varepsilon^{-1} \mathcal{E}_{\varepsilon}[u]$. Also, we will write $E_{\varepsilon}[u ; a, b]$ when the integral is over the interval $[a, b]$ instead of $[0,1]$.

In the early 1970s, Cahn and Morral [24] and DeFontaine [13] [14] initiated the study of systems of partial differential equations that model the phase separation of mixtures of three or more components in essentially the same way that the CahnHilliard equation models the separation of binary mixtures. (See Eyre [20] for a comprehensive survey of these systems.) If the domain is again taken to be $[0,1]$, then, after a change of variables, such systems can be written in the form

$$
\begin{gathered}
u_{t}=\left(-\varepsilon^{2} u_{x x}+D W(u)\right)_{x x}, \quad x \in(0,1) \\
u_{x}=u_{x x x}=0, \quad x \in\{0,1\},
\end{gathered}
$$

where $u$ is now an $n$-vector (for a mixture with $n+1$ components), and $W$ maps $\mathcal{D}(W) \subset \mathbf{R}^{n}$ into $\mathbf{R}$. Again, $\mathcal{E}_{\varepsilon}$ defined by (1.2) represents the total free energy of

\footnotetext{
* Center for Dynamical Systems and Nonlinear Studies, Georgia Institute of Technology, Atlanta, Georgia 30332. Present address, Department of Mathematics, Brigham Young University, Provo, Utah 84602 .
} 
the mixture, and it is easy to check that it provides a Lyapunov functional for (1.3). Note, also, that the mass $\int_{0}^{1} u d x$ of a solution is conserved.

We will make the following assumptions on $W$.

- $\mathcal{D}(W)$ is open, convex, and connected;

- $W \geq 0$ throughout its domain, and $W$ has only finitely many zeros, call them $\left\{z_{1}, z_{2}, \ldots, z_{m}\right\}$, (corresponding to the preferred homogeneous states, or phases, of the system);

- $W$ is $C^{3}$ on $\mathcal{D}(W)$ and has a continuous extension to its closure $\overline{\mathcal{D}(W)}$;

- The Hessian $D^{2} W$ is positive definite at each zero of $W$, and $W$ is bounded away from 0 outside of each neighborhood of these points.

Additionally, we need to require that $W$ increases as the boundary $\partial \mathcal{D}(W)$ of the domain is approached. The precise assumption we shall make is the following:

- For each point $\bar{u}$ in $\partial \mathcal{D}(W)$, there is a closed, convex set $S \subset \overline{\mathcal{D}(W)} \backslash \bar{u}$ such that

1. $W$ is nonzero on $\Omega$, the connected component of $\overline{\mathcal{D}(W)} \backslash S$ containing $\bar{u}$;

2. the function $\varphi$ that maps each point of $\mathbf{R}^{n}$ to its nearest point in $S$ satisfies $W(\varphi(u)) \leq W(u)$ for all $u \in \Omega$.

This assumption is trivially satisfied when $\mathcal{D}(W)=\mathbf{R}^{n}$. It also holds whenever $W$ is $C^{1}$ on $\overline{\mathcal{D}(W)}, \partial \mathcal{D}(W)$ is a locally compact, oriented hypersurface of class $C^{2}$, and the (exterior) normal derivative of $W$ is positive. (See, e.g., [21].) However, we state the assumption in this general way because some of the most important examples of $\mathcal{D}(W)$ do not have smooth boundaries. For example, Eyre [20] and Elliott and Luckhaus [18] study situations where $\mathcal{D}(W)$ is a convex polytope and $W$ satisfies the assumptions given above.

Note that any constant is an equilibrium solution to (1.3). A linear analysis of the equation about an unstable constant equilibrium suggests that typical solutions that start near such a constant undergo fine-grained decomposition with a characteristic length scale that is $O(\varepsilon)$. (See [22] for a precise mathematical formulation and rigorous verification of this heuristic concept in the two-component case.) This fine-grained decomposition of initially homogeneous mixtures has also been frequently observed in physical experiments [7], [9]. In this paper we investigate the way solutions evolve after this initial stage of decomposition. We, therefore, confine our attention to solutions to (1.3) with initial data $u(x, 0)=u_{0}(x)$ close to the zeros of $W$ through most of the domain, with sharp transition layers, or interfaces, separating the intervals where $u$ is nearly constant.

Consider when $n=1$ (i.e., the original Cahn-Hilliard equation (1.1)), the case for which the most work has been done. Carr, Gurtin, and Slemrod [10] showed that all of the local minimizers of $E_{\varepsilon}$ with any specified mass are monotone, so, in general, we would expect that the fine-grained structure of $u$ would coarsen as $t \rightarrow \infty$. Numerical work by Elliott and French [17] indicates that this evolution occurs very slowly. (Such slowly evolving states are sometimes said to be dynamically metastable.) Bronsard and Hilhorst [5] have shown that, in a certain space, this evolution occurs at a rate that is $O\left(\varepsilon^{k}\right)$ for any power $k$. Using completely different techniques, Alikakos, Bates, and Fusco [1] constructed a portion of the unstable manifold of a two-layer equilibrium that intersects a small neighborhood of a monotone equilibrium and showed that the speed of the flow along this connecting orbit, measured in the $H^{-1}$ norm, is $O(\exp (-C / \varepsilon))$ for some constant $C$. Recently, Bates and Xun [4] have found exponentially slow motion for the multi-layer states of (1.1) by combining the methods of [1] with those used by Carr and Pego [11] to study reaction-diffusion equations. 
The results that we present here are similar to those of Bates and Xun in that we also obtain exponentially slow motion, but the methods we use are much simpler, and they are valid not only for the two-component Cahn-Hilliard equation (1.1) but for the multi-component Cahn-Morral system (1.3), as well. It should be mentioned, however, that our results for the two-component two-layer case are weaker than those of Alikakos, Bates, and Fusco, in the sense that we do not explicitly construct heteroclinic orbits. We deal only with the speed of motion and say nothing about the geometric structure of the attractor.

In this paper, we apply the elementary, yet powerful, approach introduced by Bronsard and Kohn [6] in their study of slow motion for reaction-diffusion equations. The improvement from superpolynomial to exponential speed is made possible by incorporating some ideas of Alikakos and McKinney [2] about the profile of constrained minimizers of (1.2). Use is also made of techniques of Sternberg [27] for describing the nature of globally stable steady-state solutions of (1.3) in the limit as $\varepsilon \rightarrow 0$.

In Section 2 we present a lower bound on the energy of any function that is sufficiently close to a given simple function whose range is a subset of $W^{-1}(\{0\})$. This result amounts to an error estimate for a convergence result of Baldo [3]. In Section 3 we show how this estimate yields our main result on slow evolution of solutions with transition layers. As in [6], the only information used about the timedependent partial differential equation is the time rate of change of the energy along a solution path in phase space. Finally, in Section 4 we consider what the main result implies about the motion of the transition layers themselves.

The questions of existence and regularity of solutions for (1.1) and (1.3) have been extensively studied, and different authors have obtained various conditions on $W$ that ensure global existence of solutions [15], [16], [18], [19], [20], [25], [26], [28], [30]. Rather than restricting ourselves to one particular set of such conditions, we shall simply assume that $W$ is such that for sufficiently smooth initial data with range in $\mathcal{D}(W)$ there exists a global solution that is in $C\left(\mathbf{R}^{+} ; H^{2}(0,1)\right) \cap L^{2}\left(0, T ; H^{4}(0,1)\right)$. Given that global solutions exist, our goal is to provide some information about how some of them evolve.

2. Error Estimates. Fix $v:[0,1] \rightarrow W^{-1}(\{0\})$ having (exactly) $N$ jumps located at $\left\{x_{1}, x_{2}, \ldots, x_{N}\right\} \subset(0,1)$. Fix $r$ so small that $B\left(x_{k}, r\right) \subset[0,1]$ for each $k$, and

$$
B\left(x_{k}, r\right) \cap B\left(x_{\ell}, r\right)=\emptyset
$$

whenever $k \neq \ell$. (Here and below, $B(x, r)$ represents the open ball of radius $r$ centered at $x$ in the relevant space.) Let $\lambda_{j}$ be the minimum of the eigenvalues of $D^{2} W\left(z_{j}\right)$, and let

$$
\lambda=\min \left\{\lambda_{j}: z_{j} \in W^{-1}(\{0\})\right\} .
$$

For any function $z$ on $[0,1]$ we write $\tilde{z}(x) \equiv \int_{0}^{x} z(s) d s$. We are interested in solutions corresponding to initial data $u(x, 0)=u_{0}(x)$ such that $\tilde{u}_{0}$ is close to $\tilde{v}$ in the $L^{1}$ norm. To the discontinuous function $v$ we assign an asymptotic energy

$$
E_{0}[v] \equiv \sum_{k=1}^{N} \phi\left(v\left(x_{k}-r\right), v\left(x_{k}+r\right)\right)
$$

where

$$
\phi\left(\zeta_{1}, \zeta_{2}\right) \stackrel{\text { def }}{=} \inf \left\{J[z]: z \in A C([0,1] ; \mathcal{D}(W)), z(0)=\zeta_{1}, z(1)=\zeta_{2}\right\}
$$


and

$$
J[z] \stackrel{\text { def }}{=} \sqrt{2} \int_{0}^{1} \sqrt{W(z(s))}\left|z^{\prime}(s)\right| d s .
$$

It is easy to check that $\phi$ is a metric on the domain of $W$. Also, note that Young's inequality and a change of variable imply that

$$
E_{\varepsilon}[z ; a, b] \geq \phi(z(a), z(b)) .
$$

Lemma 2.1. Let $C$ be any positive constant less than $r \sqrt{2 \lambda}$. Then there are constants $C_{1}, \delta>0$ (depending only on $W, v$ and $C$ ) such that, for $\varepsilon$ sufficiently small,

$$
\int_{0}^{1}|\tilde{u}(x)-\tilde{v}(x)| d x \leq \delta \quad \Rightarrow \quad E_{\varepsilon}[u] \geq E_{0}[v]-C_{1} \exp (-C / \varepsilon) .
$$

Proof. Let $K$ be a compact set in the domain of $W$ containing $W^{-1}(\{0\})$ in its interior, and set $\kappa=\sup \left\{\left\|D^{3} W(\zeta)\right\|: \zeta \in K\right\}$. Choose $\hat{r}>0$ and $\rho_{1}$ so small that $C \leq(r-\hat{r}) \sqrt{2 \lambda-n \kappa \rho_{1}}$ and that $B\left(z_{j}, \rho_{1}\right)$ is contained in $K$ for each $z_{j} \in W^{-1}(\{0\})$. Choose $\rho_{2}$ so small that

$$
\begin{aligned}
& \inf \left\{\phi\left(\zeta_{1}, \zeta_{2}\right): z_{j} \in W^{-1}(\{0\}), \zeta_{1} \notin B\left(z_{j}, \rho_{1}\right), \zeta_{2} \in B\left(z_{j}, \rho_{2}\right)\right\} \\
& >\sup \left\{\phi\left(z_{j}, \zeta_{2}\right): z_{j} \in W^{-1}(\{0\}), \zeta_{2} \in B\left(z_{j}, \rho_{2}\right)\right\},
\end{aligned}
$$

and $\left|z_{j}-z_{\ell}\right|>2 \rho_{2}$ if $z_{j}$ and $z_{\ell}$ are different zeros of $W$.

Let

$$
\begin{aligned}
F\left(\rho_{2}\right)=\inf \left\{\phi\left(\zeta_{1}, \zeta_{2}\right): z_{j_{1}}, z_{j_{2}} \in W^{-1}(\{0\}), z_{j_{1}} \neq z_{j_{2}},\right. \\
\\
\left.\zeta_{1} \in B\left(z_{j_{1}}, \rho_{2}\right),\left|\left(\zeta_{2}-z_{j_{2}}\right) \cdot\left(z_{j_{2}}-z_{j_{1}}\right)\right| \leq \rho_{2}\left|z_{j_{2}}-z_{j_{1}}\right|\right\} .
\end{aligned}
$$

By our assumptions about $W, F\left(\rho_{2}\right)>0$, so there exists $M \in \mathbf{N}$ such that $M F\left(\rho_{2}\right)>$ $E_{0}[v]$. Pick such an $M$, and set $\delta=\hat{r}^{2} \rho_{2} /\left(5 M^{2}\right)$.

Now assume that $\int_{0}^{1}|\tilde{u}(x)-\tilde{v}(x)| d x \leq \delta$, and let us focus our attention on $B\left(x_{k}, r\right)$, a neighborhood of one of the transition points of $v$. For convenience, let $v_{+}=v\left(x_{k}+r\right)$ and $v_{-}=v\left(x_{k}-r\right)$. Suppose $|u-v| \geq \rho_{2}$ throughout $\left(x_{k}, x_{k}+\hat{r}\right)$, and let $I_{M}$ be an open subinterval of $\left(x_{k}, x_{k}+\hat{r}\right)$ of width $\hat{r} / M$. If we assume without loss of generality that $E_{\varepsilon}[u] \leq E_{0}[v]$ then for $\varepsilon$ sufficiently small there must be some $\hat{x} \in I_{M}$ such that $u(\hat{x}) \in B\left(z_{j_{1}}, \rho_{2}\right)$ for some $z_{j_{1}} \in W^{-1}(\{0\})$. (Otherwise the rescaled bulk free energy would be too high.) If

$$
\left|(u-v) \cdot \frac{z_{j_{1}}-v_{+}}{\left|z_{j_{1}}-v_{+}\right|}\right| \geq \rho_{2}
$$

throughout $I_{M}$ then it is not hard to check that we would have

$$
\int_{I_{M}}|\tilde{u}(x)-\tilde{v}(x)| d x \geq \int_{I_{M}}\left|(\tilde{u}(x)-\tilde{v}(x)) \cdot \frac{z_{j_{1}}-v_{+}}{\left|z_{j_{1}}-v_{+}\right|}\right| d x>\delta,
$$

which is a contradiction. Hence,

$$
\left|(u-v) \cdot \frac{z_{j_{1}}-v_{+}}{\left|z_{j_{1}}-v_{+}\right|}\right|<\rho_{2}
$$


somewhere on $I_{M}$. But then the rescaled energy on $I_{M}$ must be no less than $F\left(\rho_{2}\right)$. Partitioning $\left(x_{k}, x_{k}+\hat{r}\right)$ into $M$ equal intervals of width $\hat{r} / M$ and using the preceding result, we have $E_{\varepsilon}\left[u ; x_{k}, x_{k}+\hat{r}\right] \geq M F\left(\rho_{2}\right)>E_{0}[v]$, contrary to assumption. Hence, there is some $r_{+} \in(0, \hat{r})$ such that

$$
\left|u\left(x_{k}+r_{+}\right)-v_{+}\right|<\rho_{2} .
$$

Similarly, there is some $r_{-} \in(0, \hat{r})$ such that

$$
\left|u\left(x_{k}-r_{-}\right)-v_{-}\right|<\rho_{2} .
$$

Next, consider the unique minimizer $z:\left[x_{k}+r_{+}, x_{k}+r\right] \rightarrow \mathbf{R}^{n}$ of the functional $E_{\varepsilon}\left[z ; x_{k}+r_{+}, x_{k}+r\right]$ subject to the boundary condition

$$
z\left(x_{k}+r_{+}\right)=u\left(x_{k}+r_{+}\right) .
$$

If the range of $z$ is not contained in $B\left(v_{+}, \rho_{1}\right)$ then

$$
\begin{aligned}
E_{\varepsilon}\left[z ; x_{k}+r_{+}, x_{k}+r\right] & \geq \inf \left\{\phi\left(z\left(x_{k}+r_{+}\right), \zeta\right): \zeta \notin B\left(v_{+}, \rho_{1}\right)\right\} \\
& \geq \phi\left(z\left(x_{k}+r_{+}\right), v_{+}\right),
\end{aligned}
$$

by the choice of $\rho_{2}$ and the choice of $r_{+}$.

Suppose, on the other hand, that the range of $z$ is contained in $B\left(v_{+}, \rho_{1}\right)$. Then the Euler-Lagrange equation for $z$ is

$$
\begin{array}{cc}
z^{\prime \prime}(x)=\varepsilon^{-2} D W(z(x)), & x \in\left(x_{k}+r_{+}, x_{k}+r\right) \\
z(x)=u\left(x_{k}+r_{+}\right), & x=x_{k}+r_{+} \\
z^{\prime}(x)=0, & x=x_{k}+r .
\end{array}
$$

If we define $\psi(x) \equiv\left|z(x)-v_{+}\right|^{2}$ then $\psi^{\prime}=2\left(z-v_{+}\right) \cdot z^{\prime}$ and

$$
\psi^{\prime \prime}=2\left(\left|z^{\prime}\right|^{2}+\left(z-v_{+}\right) \cdot z^{\prime \prime}\right) \geq \frac{2}{\varepsilon^{2}}\left(z-v_{+}\right) \cdot D W(z) .
$$

Now Taylor's theorem and the choice of $\rho_{1}$ imply that

$$
D W(z)=D^{2} W\left(v_{+}\right)\left(z-v_{+}\right)+R,
$$

where $|R| \leq n \kappa\left|z-v_{+}\right|^{2} / 2$. Substituting (2.5) into (2.4) gives

$$
\begin{aligned}
\psi^{\prime \prime} & \geq \frac{2}{\varepsilon^{2}}\left(z-v_{+}\right) \cdot D^{2} W\left(v_{+}\right)\left(z-v_{+}\right)-\frac{n \kappa}{\varepsilon^{2}}\left|z-v_{+}\right|^{3} \\
& \geq \frac{2 \lambda}{\varepsilon^{2}}\left|z-v_{+}\right|^{2}-\frac{n \kappa \rho_{1}}{\varepsilon^{2}}\left|z-v_{+}\right|^{2} \\
& \geq \frac{\mu^{2}}{\varepsilon^{2}}\left|z-v_{+}\right|^{2} \\
& =\frac{\mu^{2}}{\varepsilon^{2}} \psi,
\end{aligned}
$$

where $\mu=C /(r-\hat{r})$.

Thus, $\psi$ satisfies

$$
\begin{array}{cc}
\psi^{\prime \prime}(x)-(\mu / \varepsilon)^{2} \psi(x) \geq 0, & x \in\left(x_{k}+r_{+}, x_{k}+r\right) \\
\psi(x)=\left|u\left(x_{k}+r_{+}\right)-v_{+}\right|^{2}, & x=x_{k}+r_{+} \\
\psi^{\prime}(x)=0, & x=x_{k}+r .
\end{array}
$$


Following Alikakos and McKinney [2], we compare $\psi$ to the solution $\hat{\psi}$ of

$$
\begin{array}{cc}
\hat{\psi}^{\prime \prime}(x)-(\mu / \varepsilon)^{2} \hat{\psi}(x)=0, & x \in\left(x_{k}+r_{+}, x_{k}+r\right) \\
\hat{\psi}(x)=\left|u\left(x_{k}+r_{+}\right)-v_{+}\right|^{2}, & x=x_{k}+r_{+} \\
\hat{\psi}^{\prime}(x)=0, & x=x_{k}+r,
\end{array}
$$

which can be explicitly calculated to be

$$
\hat{\psi}(x)=\frac{\left|u\left(x_{k}+r_{+}\right)-v_{+}\right|^{2}}{\cosh \left[(\mu / \varepsilon)\left(r-r_{+}\right)\right]} \cosh \left[\frac{\mu}{\varepsilon}\left(x-\left(x_{k}+r\right)\right)\right] .
$$

By the maximum principle, $\psi(x) \leq \hat{\psi}(x)$, so, in particular,

$$
\psi\left(x_{k}+r\right) \leq \frac{\left|u\left(x_{k}+r_{+}\right)-v_{+}\right|^{2}}{\cosh \left[(\mu / \varepsilon)\left(r-r_{+}\right)\right]} \leq 2 \rho_{2}^{2} \exp \left[-\frac{C}{\varepsilon}\right] .
$$

Consequently,

$$
\left|z\left(x_{k}+r\right)-v_{+}\right| \leq \rho_{2} \sqrt{2} \exp (-C /(2 \varepsilon)) .
$$

Because $W$ is quadratic at $v_{+},(2.6)$ implies that, for some constant $C_{1}$,

$$
\begin{aligned}
E_{\varepsilon}\left[z ; x_{k}+r_{+}, x_{k}+r\right] & \geq \phi\left(z\left(x_{k}+r_{+}\right), z\left(x_{k}+r\right)\right) \\
& \geq \phi\left(z\left(x_{k}+r_{+}\right), v_{+}\right)-\phi\left(v_{+}, z\left(x_{k}+r\right)\right) \\
& \geq \phi\left(z\left(x_{k}+r_{+}\right), v_{+}\right)-\left(C_{1} /(2 N)\right) \exp (-C / \varepsilon) .
\end{aligned}
$$

Combining (2.2) and (2.7), we see that the constrained minimizer of the proposed variational problem satisfies

$$
E_{\varepsilon}\left[z ; x_{k}+r_{+}, x_{k}+r\right] \geq \phi\left(z\left(x_{k}+r_{+}\right), v_{+}\right)-\left(C_{1} /(2 N)\right) \exp (-C / \varepsilon) .
$$

But the restriction of $u$ to $\left[x_{k}+r_{+}, x_{k}+r\right]$ is an admissable function, so it must satisfy the same estimate

$$
E_{\varepsilon}\left[u ; x_{k}+r_{+}, x_{k}+r\right] \geq \phi\left(u\left(x_{k}+r_{+}\right), v_{+}\right)-\left(C_{1} /(2 N)\right) \exp (-C / \varepsilon) .
$$

A similar estimate holds for the energy of $u$ on the interval $\left[x_{k}-r, x_{k}-r_{-}\right]$. Hence,

$$
\begin{aligned}
E_{\varepsilon}\left[u ; x_{k}-r, x_{k}+r\right]= & E_{\varepsilon}\left[u ; x_{k}-r, x_{k}-r_{-}\right]+E_{\varepsilon}\left[u ; x_{k}-r_{-}, x_{k}+r_{+}\right] \\
& +E_{\varepsilon}\left[u ; x_{k}+r_{+}, x_{k}+r\right] \\
\geq & \phi\left(v_{-}, u\left(x_{k}-r_{-}\right)\right)-\left(C_{1} /(2 N)\right) \exp (-C / \varepsilon) \\
& +\phi\left(u\left(x_{k}-r_{-}\right), u\left(x_{k}+r_{+}\right)\right) \\
& +\phi\left(u\left(x_{k}+r_{+}\right), v_{+}\right)-\left(C_{1} /(2 N)\right) \exp (-C / \varepsilon) \\
\geq & \phi\left(v\left(x_{k}-r\right), v\left(x_{k}+r\right)\right)-\left(C_{1} / N\right) \exp (-C / \varepsilon) .
\end{aligned}
$$

Assembling all of our estimates,

$$
E_{\varepsilon}[u] \geq \sum_{k=1}^{N} E_{\varepsilon}\left[u ; x_{k}-r, x_{k}+r\right] \geq E_{0}[v]-C_{1} \exp (-C / \varepsilon),
$$

as was claimed. 
3. Slow Evolution. In this section we will consider a family of solutions $u^{\varepsilon}(x, t)$ to (1.3), parametrized by the corresponding interaction length $\varepsilon$.

Lemma 3.1. Suppose that $C<r \sqrt{2 \lambda}$ and the initial data $u_{0}^{\varepsilon}$ satisfies

$$
\int_{0}^{1}\left|\tilde{u}_{0}^{\varepsilon}(x)-\tilde{v}(x)\right| d x \leq \frac{\delta}{2}
$$

and

$$
E_{\varepsilon}\left[u_{0}^{\varepsilon}\right] \leq E_{0}[v]+\frac{1}{g(\varepsilon)}
$$

for some function $g$ and for all $\varepsilon$ small, where $\delta$ is as in Lemma 2.1. Then

$$
\lim _{\varepsilon \rightarrow 0}\left\{\sup _{0 \leq t \leq \min \{g(\varepsilon), \exp (C / \varepsilon)\}} \int_{0}^{1}\left|\tilde{u}^{\varepsilon}(x, t)-\tilde{u}_{0}^{\varepsilon}(x)\right| d x\right\}=0 .
$$

Proof. First note that the scaled total energy $E_{\varepsilon}\left[u^{\varepsilon}(\cdot, t)\right]$ of the solution of a Cahn-Morral system is nonincreasing in $t$, since

$$
\begin{aligned}
\frac{d}{d t} E_{\varepsilon}\left[u^{\varepsilon}(\cdot, t)\right] & =\varepsilon^{-1} \int_{0}^{1}\left[D W\left(u^{\varepsilon}\right) \cdot u_{t}^{\varepsilon}+\varepsilon^{2} u_{x}^{\varepsilon} \cdot u_{x t}^{\varepsilon}\right] d x \\
& =\varepsilon^{-1} \int_{0}^{1}\left[\left(D W\left(u^{\varepsilon}\right)-\varepsilon^{2} u_{x x}^{\varepsilon}\right) \cdot u_{t}^{\varepsilon}\right] d x \\
& =-\varepsilon^{-1} \int_{0}^{1}\left|\tilde{u}_{t}^{\varepsilon}\right|^{2} d x .
\end{aligned}
$$

Integrating this equation over $t \in(0, T)$ gives

$$
E_{\varepsilon}\left[u_{0}^{\varepsilon}\right]-E_{\varepsilon}\left[u^{\varepsilon}(\cdot, T)\right]=\varepsilon^{-1} \int_{0}^{T} \int_{0}^{1}\left|\tilde{u}_{t}^{\varepsilon}\right|^{2} d x d t .
$$

Next, assume that $u_{0}^{\varepsilon}$ satisfies the conditions of the lemma and that $T$ is small enough that

$$
\int_{0}^{T} \int_{0}^{1}\left|\tilde{u}_{t}^{\varepsilon}\right| d x d t \leq \delta / 2
$$

Then

$$
\int_{0}^{1}\left|\tilde{u}_{0}^{\varepsilon}(x)-\tilde{u}^{\varepsilon}(x, T)\right| d x \leq \delta / 2,
$$

so by the triangle inequality,

$$
\int_{0}^{1}\left|\tilde{u}^{\varepsilon}(x, T)-\tilde{v}(x)\right| d x \leq \delta .
$$

Applying, Lemma 2.1 to $\tilde{u}^{\varepsilon}(\cdot, T)$ gives $E_{\varepsilon}\left[u^{\varepsilon}(\cdot, T)\right] \geq E_{0}[v]-C_{1} \exp (-C / \varepsilon)$. In combination with (3.2), this yields

$$
\begin{aligned}
\int_{0}^{T} \int_{0}^{1}\left|\tilde{u}_{t}^{\varepsilon}\right|^{2} d x d t & =\varepsilon\left(E_{\varepsilon}\left[u_{0}^{\varepsilon}\right]-E_{\varepsilon}\left[u^{\varepsilon}(\cdot, T)\right]\right) \\
& \leq C_{1} \varepsilon\left[\frac{1}{g(\varepsilon)}+\exp (-C / \varepsilon)\right]
\end{aligned}
$$


assuming, without loss of generality, that $C_{1} \geq 1$.

Using Hölder's inequality and (3.3) we have

$$
\begin{aligned}
\left(\int_{0}^{T} \int_{0}^{1}\left|\tilde{u}_{t}^{\varepsilon}\right| d x d t\right)^{2} & \leq\left(\int_{0}^{T} \int_{0}^{1} 1 d x d t\right) \cdot\left(\int_{0}^{T} \int_{0}^{1}\left|\tilde{u}_{t}^{\varepsilon}\right|^{2} d x d t\right) \\
& \leq C_{1} T \varepsilon\left[\frac{1}{g(\varepsilon)}+\exp (-C / \varepsilon)\right] .
\end{aligned}
$$

Hence,

$$
T \geq \frac{1}{C_{1} \varepsilon}\left[\frac{1}{g(\varepsilon)}+\exp (-C / \varepsilon)\right]^{-1}\left(\int_{0}^{T} \int_{0}^{1}\left|\tilde{u}_{t}^{\varepsilon}\right| d x d t\right)^{2} .
$$

Now suppose that

$$
\int_{0}^{\infty} \int_{0}^{1}\left|\tilde{u}_{t}^{\varepsilon}\right| d x d t \geq \delta / 2
$$

Then we can choose $T$ such that $\int_{0}^{T} \int_{0}^{1}\left|\tilde{u}_{t}^{\varepsilon}\right| d x d t=\delta / 2$. For this choice of $T$, (3.4) yields

$$
T \geq \frac{\delta^{2}}{4 C_{1} \varepsilon\left[\frac{1}{g(\varepsilon)}+\exp (-C / \varepsilon)\right]} \geq \frac{\delta^{2}}{8 C_{1} \varepsilon} \min \{g(\varepsilon), \exp (C / \varepsilon)\} .
$$

Then (3.3) implies that

$$
\int_{0}^{\delta^{2} \min \{g(\varepsilon), \exp (C / \varepsilon)\} /\left(8 C_{1} \varepsilon\right)} \int_{0}^{1}\left|\tilde{u}_{t}^{\varepsilon}\right|^{2} d x d t \leq C_{1} \varepsilon\left[\frac{1}{g(\varepsilon)}+\exp (-C / \varepsilon)\right] .
$$

If, on the other hand, $\int_{0}^{\infty} \int_{0}^{1}\left|\tilde{u}_{t}^{\varepsilon}\right| d x d t<\delta / 2$, then (3.3) must hold for every $T$; therefore, (3.5) is also true for this case.

Using Hölder's inequality and (3.5) we see that for $\varepsilon<\delta^{2} /\left(8 C_{1}\right)$

$$
\begin{aligned}
& \sup _{0 \leq t \leq \min \{g(\varepsilon), \exp (C / \varepsilon)\}} \int_{0}^{1}\left|\tilde{u}^{\varepsilon}(x, t)-\tilde{u}_{0}^{\varepsilon}(x)\right| d x \\
& \leq \int_{0}^{\min \{g(\varepsilon), \exp (C / \varepsilon)\}} \int_{0}^{1}\left|\tilde{u}_{t}^{\varepsilon}\right| d x d t \\
& \leq\left(\min \{g(\varepsilon), \exp (C / \varepsilon)\} \int_{0}^{\min \{g(\varepsilon), \exp (C / \varepsilon)\}} \int_{0}^{1}\left|\tilde{u}_{t}^{\varepsilon}\right|^{2} d x d t\right)^{1 / 2} \\
& \leq\left(\min \{g(\varepsilon), \exp (C / \varepsilon)\} C_{1} \varepsilon\left[\frac{1}{g(\varepsilon)}+\exp (-C / \varepsilon)\right]\right)^{1 / 2} \\
& \leq \sqrt{2 C_{1} \varepsilon} .
\end{aligned}
$$

Letting $\varepsilon \rightarrow 0$ we get (3.1).

The strength of estimate (3.1) in Lemma 3.1 depends on the efficiency of the transition layers in the initial data. In Theorem 3.3 below, we show that, in a neighborhood of the step function $v$, there exist initial data that smooth out the discontinuities of $v$ 
in an efficient enough manner that the corresponding solutions of (1.3) evolve exponentially slowly. Before we present this theorem, we shall state and prove a technical lemma about the existence and regularity of minimizing geodesics for the degenerate Riemannian metric $\phi$.

LEMMA 3.2.

1. For any two zeros $z_{i}$ and $z_{j}$ of $W$, there is a Lipschitz continuous path $\gamma_{i j}$ from $z_{i}$ to $z_{j}$, parametrized by a multiple of Euclidean arclength, that realizes the distance $\phi\left(z_{i}, z_{j}\right)$; i.e., $\phi\left(z_{i}, z_{j}\right)=J\left[\gamma_{i j}\right]$.

2. There exists a positive constant $C_{2}$ such that $\left|\gamma_{i j}(y)-z_{i}\right| \geq C_{2} y$ for $y$ sufficiently small, and $\left|\gamma_{i j}(y)-z_{j}\right| \geq C_{2}(1-y)$ for $y$ sufficiently near 1 .

Proof. Recall that outside of a neighborhood of its zeros $W$ is bounded away from 0 ; therefore, it is possible to find a bounded set $B \subset \mathcal{D}(W)$ such that if $\gamma(0)=z_{i}$, $\gamma(1)=z_{j}$, and $J[\gamma] \leq \phi\left(z_{i}, z_{j}\right)+1$ then the image of $\gamma$ is contained in $B$. Extend $W$ continuously to $\bar{B}$, and consider the problem of minimizing $J[\gamma]$ over all $\gamma$ satisfying these boundary conditions and having images contained in $\bar{B}$. Now, $J[\gamma]$ is a parametric integral, and it is known that this new minimization problem has an $A C$ global minimizer $\gamma_{i j}$ [12]. The parameter of this minimizer can be chosen to be proportional to arclength, and then $\gamma_{i j}$ will be Lipschitz continuous.

We claim that $\gamma_{i j}([0,1])$ is contained in $\mathcal{D}(W)$. Suppose it is not. Then there exists some $y \in(0,1)$ such that $\gamma_{i j}(y) \in \partial \mathcal{D}(W)$. By the assumptions on $W$, there is a closed, convex set $S \subset \overline{\mathcal{D}(W)} \backslash \gamma_{i j}(y)$ such that $W$ is nonzero on the connected component $\Omega$ of $\overline{\mathcal{D}(W)} \backslash S$ containing $\gamma_{i j}(y)$, and the function $\varphi$ that maps each point of $\mathbf{R}^{n}$ to its nearest point in $S$ satisfies $W(\varphi(u)) \leq W(u)$ for all $u$ in $\Omega$. Consider the modified path $\bar{\gamma}_{i j}$ from $z_{i}$ to $z_{j}$ defined by

$$
\bar{\gamma}_{i j}(y)= \begin{cases}\varphi\left(\gamma_{i j}(y)\right), & \text { if } \gamma_{i j}(y) \in \Omega \\ \gamma_{i j}(y), & \text { otherwise }\end{cases}
$$

Note that $\varphi$ is Lipschitz continuous with Lipschitz constant 1. Because of this and the fact that $S$ separates $\Omega$ from the rest of $\overline{\mathcal{D}(W)}, \bar{\gamma}_{i j}$ is Lipschitz continuous. It is also easy to check that $J\left[\bar{\gamma}_{i j}\right]<J\left[\gamma_{i j}\right]$. This contradicts the optimality of $\gamma_{i j}$; hence, the claim holds. This verifies that $\phi\left(z_{i}, z_{j}\right)=J\left[\gamma_{i j}\right]$.

We now prove the estimate on $\gamma_{i j}$ near $z_{i}$; the estimate near $z_{j}$ can be derived similarly. Again, we consider a modification of $\gamma_{i j}$, this time the path $\gamma_{i j}^{\eta}$ defined by

$$
\gamma_{i j}^{\eta}(y)=\left\{\begin{array}{ll}
z_{i}+(y / \eta)\left(\gamma_{i j}(\eta)-z_{i}\right), & \text { if } 0 \leq y \leq \eta \\
\gamma_{i j}(y), & \text { otherwise }
\end{array} .\right.
$$

The optimality of $\gamma_{i j}$ implies that

$$
\sqrt{2} \int_{0}^{\eta} \sqrt{W\left(\gamma_{i j}(s)\right)}\left|\gamma_{i j}^{\prime}(s)\right| d s \leq \sqrt{2} \int_{0}^{\eta} \sqrt{W\left(\gamma_{i j}^{\eta}(s)\right)} \frac{\left|\gamma_{i j}(\eta)-z_{i}\right|}{\eta} d s .
$$

Because $D^{2} W\left(z_{i}\right)$ is positive definite, there are positive constants $M_{1}$ and $M_{2}$ such that

$$
M_{1}\left|u-z_{i}\right| \leq \sqrt{W(u)} \leq M_{2}\left|u-z_{i}\right|
$$

in a small neighborhood of $z_{i}$. Using this in (3.6), we find that

$$
\left|\gamma_{i j}(\eta)-z_{i}\right|^{2} \geq M_{3} \int_{0}^{\eta}\left|\gamma_{i j}(s)-z_{i}\right| d s
$$


for some constant $M_{3}$. Applying a variant of Gronwall's inequality [23] we obtain the desired estimate.

THEOREM 3.3. Given $\delta>0$, there exist constants $C, \hat{\varepsilon}>0$ and a family of initial conditions $\left\{u_{0}^{\varepsilon}: 0 \leq \varepsilon \leq \hat{\varepsilon}\right\}$ of (1.3) satisfying homogeneous Neumann boundary conditions and the estimate

$$
\int_{0}^{1}\left|\tilde{u}_{0}^{\varepsilon}(x)-\tilde{v}(x)\right| d x \leq \frac{\delta}{2}
$$

such that the corresponding solutions $u^{\varepsilon}$ of (1.3) satisfy

$$
\lim _{\varepsilon \rightarrow 0}\left\{\sup _{0 \leq t \leq \exp (C / \varepsilon)} \int_{0}^{1}\left|\tilde{u}^{\varepsilon}(x, t)-\tilde{u}_{0}^{\varepsilon}(x)\right| d x\right\}=0 .
$$

Proof. Lemma 3.2 shows that to each discontinuity $x_{k}$ of $v$ there corresponds an optimal path connecting $v\left(x_{k}-r\right)$ to $v\left(x_{k}+r\right)$. Note that it suffices to prove the present theorem under the assumption that none of these optimal paths passes through any zero of $W$ (except at the endpoints of the path), since if the assumption is not satisfied then $v$ can be perturbed slightly to create a new step function that does satisfy the assumption.

Given $\varepsilon$, set $u_{0}^{\varepsilon}=v$ outside of $\cup_{j=1}^{m} B\left(x_{k}, r\right)$. For fixed $x_{k}$, we shall again use the notation $v_{ \pm}$for $v\left(x_{k} \pm r\right)$ and will show that for $\varepsilon$ sufficiently small we can define $u_{0}^{\varepsilon}$ inside $B\left(x_{k}, r\right)$ in such a way that $u_{0}^{\varepsilon}$ is very close to $v$ (in the $L^{1}$ sense) on $B\left(x_{k}, r\right)$, $E_{\varepsilon}\left[u_{0}^{\varepsilon} ; x_{k}-r, x_{k}+r\right] \leq \phi\left(v_{-}, v_{+}\right)+C_{3} \exp (-C / \varepsilon)$ for some $C$ and $C_{3}$, and $u_{0}^{\varepsilon}$ is continuous at the endpoints of $B\left(x_{k}, r\right)$. By taking $C$ slightly smaller and applying Lemma 3.1, the proof of the theorem will then be complete.

Let $\gamma:[0,1] \rightarrow \mathbf{R}^{n}$ be an optimal path from $v_{-}$to $v_{+}$as described in Lemma 3.2. Let $\sigma$ be the Euclidean arclength of $\gamma$. Let $y: \mathbf{R} \rightarrow[0,1]$ be the solution of

$$
\frac{d y}{d \xi}=\sigma^{-1} \sqrt{2 W(\gamma(y(\xi)))}
$$

satisfying $y(0)=1 / 2$. (Since $\sqrt{W}$ and $\gamma$ are Lipschitz continuous, a unique $C^{1}$ solution is guaranteed to exist.) Note that $\lim _{\xi \rightarrow \infty} y(\xi)=1$ and $\lim _{\xi \rightarrow-\infty} y(\xi)=0$. Define $u_{0}^{\varepsilon}$ inside $B\left(x_{k}, r\right)$ by

$$
u_{0}^{\varepsilon}(x)=\left\{\begin{array}{cc}
v_{-}+\left(\gamma(y(1-r / \varepsilon))-v_{-}\right)\left(x-x_{k}+r\right) / \varepsilon, & x_{k}-r<x<x_{k}-r+\varepsilon \\
\gamma\left(y\left(\left(x-x_{k}\right) / \varepsilon\right)\right), & x_{k}-r+\varepsilon \leq x \leq x_{k}+r-\varepsilon \\
v_{+}+\left(v_{+}-\gamma(y(r / \varepsilon-1))\right)\left(x-x_{k}-r\right) / \varepsilon, & x_{k}+r-\varepsilon<x<x_{k}+r .
\end{array}\right.
$$

It is easy to see that $u_{0}^{\varepsilon}$ is continuous and, for $\varepsilon$ sufficiently small, will satisfy the $L^{1}$ requirement; therefore, we only need to check the energy requirement. Note that

$$
\begin{aligned}
E_{\varepsilon}\left[u_{0}^{\varepsilon} ; x_{k}-r, x_{k}+r\right]= & \int_{-r}^{-r+\varepsilon}\left[\frac{1}{\varepsilon} W\left(u_{0}^{\varepsilon}\left(x+x_{k}\right)\right)+\frac{\varepsilon}{2}\left|u_{0}^{\varepsilon^{\prime}}\left(x+x_{k}\right)\right|^{2}\right] d x \\
& +\int_{-r+\varepsilon}^{r-\varepsilon}\left[\frac{1}{\varepsilon} W\left(u_{0}^{\varepsilon}\left(x+x_{k}\right)\right)+\frac{\varepsilon}{2}\left|u_{0}^{\varepsilon \prime}\left(x+x_{k}\right)\right|^{2}\right] d x \\
& +\int_{r-\varepsilon}^{r}\left[\frac{1}{\varepsilon} W\left(u_{0}^{\varepsilon}\left(x+x_{k}\right)\right)+\frac{\varepsilon}{2}\left|u_{0}^{\varepsilon \prime}\left(x+x_{k}\right)\right|^{2}\right] d x \\
\stackrel{\text { def }}{=} & I_{1}+I_{2}+I_{3} .
\end{aligned}
$$


Now, using (3.7) and the definition of $\gamma$ we have

$$
\begin{aligned}
I_{2} & =\int_{-r+\varepsilon}^{r-\varepsilon}\left[\frac{1}{\varepsilon} W\left(\gamma\left(y\left(\frac{x}{\varepsilon}\right)\right)\right)+\frac{1}{2 \varepsilon}\left|\gamma^{\prime}\left(y\left(\frac{x}{\varepsilon}\right)\right) y^{\prime}\left(\frac{x}{\varepsilon}\right)\right|^{2}\right] d x \\
& =\int_{1-r / \varepsilon}^{r / \varepsilon-1}\left[W(\gamma(y(\xi)))+\frac{1}{2}\left|\gamma^{\prime}(y(\xi)) y^{\prime}(\xi)\right|^{2}\right] d \xi \\
& =\int_{1-r / \varepsilon}^{r / \varepsilon-1} \sqrt{2 W(\gamma(y(\xi)))}\left|\gamma^{\prime}(y(\xi))\right| y^{\prime}(\xi) d \xi \\
& =\int_{y(1-r / \varepsilon)}^{y(r / \varepsilon-1)} \sqrt{2 W(\gamma(y))}\left|\gamma^{\prime}(y)\right| d y \\
& \leq \phi\left(v_{-}, v_{+}\right) .
\end{aligned}
$$

Next, we estimate $I_{1}$ (letting $C_{3}$ represent a constant whose value may change from line to line):

$$
\begin{aligned}
I_{1}= & \frac{1}{\varepsilon} \int_{-r}^{-r+\varepsilon} W\left(v_{-}+\frac{\gamma(y(1-r / \varepsilon))-v_{-}}{\varepsilon}(x+r)\right) d x \\
& +\frac{1}{2}\left|\gamma\left(y\left(1-\frac{r}{\varepsilon}\right)\right)-v_{-}\right|^{2} \\
\leq & C_{3}\left|\gamma\left(y\left(1-\frac{r}{\varepsilon}\right)\right)-v_{-}\right|^{2} \\
\leq & C_{3}\left(y\left(1-\frac{r}{\varepsilon}\right)\right)^{2} .
\end{aligned}
$$

Now, Lemma 3.2 implies that there exists a constant $C>0$ such that, for $\xi \ll 0$,

$$
\begin{aligned}
y^{\prime}(\xi) & =\sigma^{-1} \sqrt{2 W(\gamma(y(\xi))} \\
& \geq \frac{C}{2 r C_{2}}\left|\gamma(y(\xi))-v_{-}\right| \\
& \geq \frac{C}{2 r}(y(\xi)) .
\end{aligned}
$$

Applying a simple comparison argument to (3.11) yields

$$
y(\xi) \leq C_{3} \exp \left(\frac{C \xi}{2 r}\right),
$$

for $\xi \ll 0$. Substituting this into (3.10) we have

$$
I_{1} \leq C_{3} \exp (-C / \varepsilon)
$$

Similarly,

$$
I_{3} \leq C_{3} \exp (-C / \varepsilon) .
$$

By substituting (3.9), (3.12), and (3.13) into (3.8), we see that $u_{0}^{\varepsilon}$ satisfies the energy requirement, so we are done.

REMARK. For the standard two-component case with $W$ having two minima, the maximum principle can be used more directly in the proof of Lemma 2.1 (see [2]) 
and an explicit value of $C$ can be obtained in Theorem 3.3. This $C$ agrees with that obtained in [1] and [4].

Remark. The initial data $u_{0}^{\varepsilon}$ just constructed are in $W^{1, \infty}(0,1)$. Since $E_{\varepsilon}$ is continuous on this space and elements of this space can be approximated arbitrarily closely by $C^{p}$ functions (for arbitrarily large $p$ ), the initial data in Theorem 3.3 can be assumed to be arbitrarily smooth.

4. Motion of Transition Layers. From Theorem 3.3, which establishes slow evolution in a certain abstract space, it is natural to infer that the transition layers themselves move extremely slowly. This concept can be made precise in a number of ways, one of which we present here.

Fix some closed subset $K$ of $\mathcal{D}(W) \backslash W^{-1}(\{0\})$, and define the interface $I[u]$ of a function $u$ by

$$
I[u] \stackrel{\text { def }}{=} u^{-1}(K)
$$

This terminology is natural, since the set $K$ is bounded away from the phases of $W$, where the bulk energy is low. By analyzing how rapidly $I[u]$ changes, we obtain information on how fast the transition layers move.

Let $d(A, B)$ denote the Hausdorff distance between the sets $A$ and $B$, i.e.

$$
d(A, B)=\max \left\{\sup _{a \in A} d(a, B), \sup _{b \in B} d(b, A)\right\} .
$$

We shall show that $d\left(I\left[u^{\varepsilon}(\cdot, t)\right], I\left[u_{0}^{\varepsilon}\right]\right)$ grows very slowly in $t$.

THEOREM 4.1. Fix $\hat{r}>0$ and $\hat{\delta}>0$. Then there exist constants $C, \hat{\varepsilon}>0$ and $a$ family of initial conditions $\left\{u_{0}^{\varepsilon}: 0 \leq \varepsilon \leq \hat{\varepsilon}\right\}$ of (1.3) satisfying homogeneous Neumann boundary conditions and the estimate

$$
\int_{0}^{1}\left|\tilde{u}_{0}^{\varepsilon}(x)-\tilde{v}(x)\right| d x \leq \hat{\delta}
$$

such that the time $T(\hat{r})$ necessary for $d\left(I\left[u^{\varepsilon}(\cdot, T(\hat{r}))\right], I\left[u_{0}^{\varepsilon}\right]\right)$ to exceed $\hat{r}$ satisfies

$$
T(\hat{r}) \geq \exp (C / \varepsilon)
$$

Proof. Assume, without loss of generality, that $\hat{r} \leq r$. Choose $\hat{\rho}$ small enough that

$$
\begin{gathered}
\inf \left\{\phi\left(\zeta_{1}, \zeta_{2}\right): z_{j} \in W^{-1}(\{0\}), \zeta_{1} \in K, \zeta_{2} \in B\left(z_{j}, \hat{\rho}\right)\right\} \\
>4 N \sup \left\{\phi\left(z_{j}, \zeta_{2}\right): z_{j} \in W^{-1}(\{0\}), \zeta_{2} \in B\left(z_{j}, \hat{\rho}\right)\right\} .
\end{gathered}
$$

Choose $M \in \mathbf{N}$ so large that $M F(\hat{\rho})>E_{0}[v]$, where $F$ is defined as in (2.1).

We claim that there exists $\varepsilon_{0}>0$ such that for all $\varepsilon \leq \varepsilon_{0}$ and for all functions $z:[0,1] \rightarrow \mathbf{R}^{n}$ satisfying

$$
\int_{0}^{1}|\tilde{z}(x)-\tilde{v}(x)| d x \leq \frac{\hat{\rho} \hat{r}^{2}}{17 M^{2}}
$$

and

$$
E_{\varepsilon}[z] \leq E_{0}[v]+2 N \sup \left\{\phi\left(z_{j}, \zeta_{2}\right): z_{j} \in W^{-1}(\{0\}), \zeta_{2} \in B\left(z_{j}, \hat{\rho}\right)\right\},
$$


we have

$$
d\left(I[z],\left\{x_{k}\right\}_{k=1}^{N}\right)<\frac{\hat{r}}{2} .
$$

Verification of Claim: Note first that if $\varepsilon$ is sufficiently small then for each $k$ there exist

$$
x_{k-} \in\left(x_{k}-\hat{r} / 2, x_{k}\right)
$$

and

$$
x_{k+} \in\left(x_{k+}, x_{k}+\hat{r} / 2\right)
$$

such that $\left|z\left(x_{k \pm}\right)-v\left(x_{k \pm}\right)\right|<\hat{\rho}$. This follows as in the proof of Lemma 2.1. Now, suppose the claim is violated. Then, reasoning as before

$$
\begin{aligned}
& E_{\varepsilon}[z] \geq \sum_{k=1}^{N} E_{\varepsilon}\left[z ; x_{k-}, x_{k+}\right] \\
& \quad+\inf \left\{\phi\left(\zeta_{1}, \zeta_{2}\right): z_{j} \in W^{-1}(\{0\}), \zeta_{1} \in K, \zeta_{2} \in B\left(z_{j}, \hat{\rho}\right)\right\} \\
& \geq E_{0}[v]-2 N \sup \left\{\phi\left(z_{j}, \zeta_{2}\right): z_{j} \in W^{-1}(\{0\}), \zeta_{2} \in B\left(z_{j}, \hat{\rho}\right)\right\} \\
& \quad+\inf \left\{\phi\left(\zeta_{1}, \zeta_{2}\right): z_{j} \in W^{-1}(\{0\}), \zeta_{1} \notin K, \zeta_{2} \in B\left(z_{j}, \hat{\rho}\right)\right\} \\
& >E_{0}[v]+2 N \sup \left\{\phi\left(z_{j}, \zeta_{2}\right): z_{j} \in W^{-1}(\{0\}), \zeta_{2} \in B\left(z_{j}, \hat{\rho}\right)\right\} \\
& \geq E_{\varepsilon}[z],
\end{aligned}
$$

which is a contradiction. Thus, the claim is true.

Apply Theorem 3.3 with $\delta=\min \left\{\hat{\delta}, \hat{\rho} \hat{r}^{2} /\left(17 M^{2}\right)\right.$ to obtain a parametrized set of initial conditions $\left\{u_{0}^{\varepsilon}: 0 \leq \varepsilon \leq \hat{\varepsilon}\right\}$. Note that $z=u_{0}^{\varepsilon}$ satisfies (4.2) and, by the construction in the proof of Theorem 3.3, satisfies (4.3) for $\varepsilon$ sufficiently small. Applying the claim we get

$$
d\left(I\left[u_{0}^{\varepsilon}\right],\left\{x_{k}\right\}_{k=1}^{N}\right)<\frac{\hat{r}}{2},
$$

for $\varepsilon$ sufficiently small. By Theorem 3.3, the triangle inequality, and the fact that $E_{\varepsilon}\left[u^{\varepsilon}(\cdot, t)\right]$ is decreasing in $t$, we see that there is a constant $C>0$ such that for $\varepsilon$ sufficiently small, $z=u^{\varepsilon}(\cdot, T)$ satisfies (4.2) and (4.3) if $T \leq \exp (C / \varepsilon)$. Thus, for all such $T$ we also have

$$
d\left(I\left[u^{\varepsilon}(\cdot, T)\right],\left\{x_{k}\right\}_{k=1}^{N}\right)<\frac{\hat{r}}{2} .
$$

By the triangle inequality we get

$$
d\left(I\left[u_{0}^{\varepsilon}\right], I\left[u^{\varepsilon}(\cdot, T)\right]\right)<\hat{r} .
$$

This means that (4.1) must hold.

Acknowledgments. I am indebted to K. Mischaikow for suggesting this problem and to P. Bates for helping to correct a mistake in the original manuscript. I would also like to thank the referees for their helpful suggestions. 


\section{REFERENCES}

[1] N. D. Alikakos, P. W. Bates, and G. Fusco, Slow motion for the Cahn-Hilliard equation in one space dimension, J. Differential Equations, 90 (1991), pp. 81-135.

[2] N. D. Alikakos And W. R. McKinney, Remarks on the equilibrium theory for the CahnHilliard equation in one space dimension, in Reaction-Diffusion Equations, Oxford University Press, 1990.

[3] S. BALDO, Minimal interface criterion for phase transitions in mixtures of Cahn-Hilliard fluids, Ann. Inst. H. Poincaré, Analyse Nonlinèaire, 7 (1990), pp. 67-90.

[4] P. W. Bates and J. P. Xun, Metastable patterns for the Cahn-Hilliard equation, J. Differential Equations, to appear.

[5] L. Bronsard and D. Hilhorst, On the slow dynamics for the Cahn-Hilliard equation in one space dimension, Proc. Roy. Soc. London, Series A, 439 (1992), pp. 669-682.

[6] L. Bronsard AND R. V. Kohn, On the slowness of phase boundary motion in one space dimension, Comm. P. A. Math., 43 (1990), pp. 983-998.

[7] E. P. Butler and G. Thomas, Structure and properties of spinodally decomposed $\mathrm{Cu}$ - $\mathrm{Ni}$ - $\mathrm{Fe}$ alloys, Acta Metall., 18 (1970), pp. 347-365.

[8] J. W. CAhn, On spinodal decomposition, Acta Metall., 9 (1961), pp. 795-801.

[9] - Spinodal decomposition, Trans. Metallurg. Soc. of AIME, 242 (1968), pp. 166-180.

[10] J. Carr, M. E. Gurtin, And M. Slemrod, Structured phase transitions on a finite interval, Arch. Rational Mech. Anal., 86 (1984), pp. 317-351.

[11] J. Carr and R. L. Pego, Metastable patterns in solutions of $u_{t}=\varepsilon^{2} u_{x x}-f(u)$, Comm. P. A. Math., 42 (1989), pp. 523-576.

[12] L. Cesari, Optimization - Theory and Applications, vol. 17 of Applications of Mathematics, Springer-Verlag, New York, 1983.

[13] D. DeFontaine, An analysis of clustering and ordering in multicomponent solid solutions - I. stability criteria, J. Phys. Chem. Solids, 33 (1972), pp. 297-310.

[14] - An analysis of clustering and ordering in multicomponent solid solutions - II. fluctuations and kinetics, J. Phys. Chem. Solids, 34 (1973), pp. 1285-1304.

[15] T. DLotko, Fourth order semilinear parabolic equations, Tsukuba J. Math., 16 (1992), pp. 389406.

[16] C. M. ELLIOTT, The Cahn-Hilliard model for the kinetics of phase separation, in Mathematical Models for Phase Change Problems, J. F. Rodrigues, ed., Birkhäuser Verlag, Basel, 1989, pp. 35-73.

[17] C. M. Elliott And D. A. French, Numerical studies of the Cahn-Hilliard equation for phase separation, IMA J. Appl. Math., 38 (1987), pp. 97-128.

[18] C. M. Elliott and S. Luckhaus, A generalised diffusion equation for phase separation of a multi-component mixture with interfacial free energy, preprint.

[19] C. M. Elliott And S. Zheng, On the Cahn-Hilliard equation, Arch. Rational Mech. Anal., 96 (1986), pp. 339-357.

[20] D. J. Eyre, Systems of Cahn-Hilliard equations, SIAM J. Appl. Math., 53 (1993), pp. 16861712 .

[21] G. B. Folland, Introduction to Partial Differential Equations, vol. 17 of Mathematical Notes, Princeton University Press, Princeton, New Jersey, 1976.

[22] C. P. Grant, Spinodal decomposition for the Cahn-Hilliard equation, Comm. in P.D.E., 18 (1993), pp. 453-490.

[23] P. Hartman, Ordinary Differential Equations, Birkhäuser, Boston, second ed., 1982

[24] J. E. Morral and J. W. Cahn, Spinodal decomposition in ternary systems, Acta Metall., 19 (1971), pp. 1037-1045.

[25] K. Promislow, Time analyticity and Gevrey regularity for solutions of a class of dissipative partial differential equations, Nonlinear Anal., 16 (1991), pp. 959-980.

[26] S. M. RANkin, Semilinear evolution equations in Banach spaces with application to parabolic partial differential equations, Trans. Amer. Math. Soc., 336 (1993), pp. 523-536.

[27] P. Sternberg, The effect of a singular perturbation on nonconvex variational problems, Arch. Rational Mech. Anal., 101 (1988), pp. 209-260.

[28] R. Temam, Infinite-Dimensional Dynamical Systems in Mechanics and Physics, vol. 68 of Applied Mathematical Sciences, Springer-Verlag, New York, 1988.

[29] J. D. VAN DER WAALS, The thermodynamic theory of capillarity flow under the hypothesis of a continuous variation in density, Verhandel. Konink. Akad. Weten. Amsterdam, 1 (1893).

[30] S. Zheng, Asymptotic behavior of solution to the Cahn-Hilliard equation, Appl. Anal., 23 (1986), pp. 165-184. 